\title{
Correlation between amniotic fluid lamellar body concentrations at different gestational ages and respiratory distress syndrome
}

\author{
Amruta C. C.*, Hema A. Patil \\ Department of Obstetrics and Gynecology, SDM College of Medical Sciences, Dharwad, India \\ Received: 29 October 2018 \\ Accepted: 28 November 2018 \\ *Correspondence: \\ Dr. Amruta C. C., \\ E-mail: meetdramruta@gmail.com \\ Copyright: (C) the author(s), publisher and licensee Medip Academy. This is an open-access article distributed under \\ the terms of the Creative Commons Attribution Non-Commercial License, which permits unrestricted non-commercial \\ use, distribution, and reproduction in any medium, provided the original work is properly cited.
}

\begin{abstract}
Background: Respiratory distress syndrome (RDS) also known as hyaline membrane disease is the most common cause of respiratory failure in neonates. The risk of RDS rises in prematurity due to decreased production of surfactant. Lamellar bodies (LB) are storage form of surfactant and are actively secreted into the alveolar space and hence into the amniotic fluid. The objective of the present study was to find out correlation between amniotic fluid lamellar body concentrations (LBC) at different gestational ages and respiratory distress syndrome

Methods: The study was a prospective observational study conducted over a period of 1 year at department of OBG, SDM college of medical sciences, Dharwad. All women undergoing caesarean section were included in the study. 5 $\mathrm{ml}$ of amniotic fluid was collected at the time of Caesarean section and sent to laboratory, Lamellar body concentrations (LBC) was counted in auto analyzer by platelet impedance counting. Incidence of RDS at different gestational age with an LBC cut off 40,500 was calculated.

Results: Among 300 patients studied, Respiratory distress was seen in 116 (41.3\%) of patients. LBC cut off of 40,500 was chosen. Out of 140 preterm babies, 121 (86.8\%) had lamellar body count less than 40,500 and 19 (13.5\%) had more than 40,500. There is significant correlation between decreasing lamellar body count in preterms and increasing incidence of RDS.

Conclusions: LBC count increases with increasing gestational ages and is inversely proportional to the incidence of RDS in preterm newborns.
\end{abstract}

Keywords: Lamellar body counts, Preterm, RDS

\section{INTRODUCTION}

Respiratory distress syndrome (RDS) also known as hyaline membrane disease is the most common cause of respiratory failure in neonates. ${ }^{1}$ The risk of RDS rises in prematurity due to decreased production of surfactant. Respiratory distress syndrome is a major cause of morbidity and mortality in preterm neonates. With the advent of modern newborn care, many pregnancies are terminated before term in many complicated cases. These conditions warrant the assessment of lung maturity in fetus as RDS is a major cause of morbidity in preterm neonates. Lamellar bodies (LB) are storage form of surfactant within type II pneumocytes and are actively secreted into the alveolar space and hence into the amniotic fluid. They are secreted from 28 weeks of gestation; its concentration increases as the gestational age increases. ${ }^{2}$

The surfactant dispersed into the alveolar space has three different cycles: recycling, degradation and elimination. During recycling, the surfactant components are reused by the type-II cells and incorporated again in the lamellar bodies. Alternatively, the surfactant can be disintegrated, and its components reused for the synthesis of new lipids and proteins in type-II cells or eliminated from the 
system in the form of an intact molecule or a degradational product such as fatty acids. ${ }^{3}$ Once secreted, lamellar bodies become hydrated in the surface water layer and unravel to form tubular myelin, a lattice-like structure composed of lipids and proteins that support the surfactant monolayer. Fetal breathing movements in utero expel pulmonary lamellar bodies and surfactant into the amniotic fluid. Surfactant and lamellar bodies appear in the amniotic fluid at 28 to 32 weeks and increase exponentially as gestation continues. ${ }^{4}$

Lamellar body count is a new promising test in prediction of RDS in newborns. Lamellar bodies are similar in size to that of platelet and can be easily counted in platelet counting chamber of auto analysers. In the current study lamellar body count was calculated from amniotic fluid during caesarean section. Incidence of RDS in newborns were studied and an attempt was made to correlate LBC at different gestational ages and RDS. The objective of this study was to find out correlation between amniotic fluid lamellar body concentrations (LBC) at different gestational ages and respiratory distress syndrome.

\section{METHODS}

The present study was a prospective observational study conducted in department of Obstetrics and Gynecology, SDM College of medical sciences, Dharwad. It was conducted over a period of 1 year. Cases were divided into 2 groups term and preterm. Gestational age of more than 37 completed weeks till 40 weeks was term. Preterm group consisted of pregnant women with gestational age between 28 weeks to less than 37 completed weeks.

Ethical committee clearance was obtained from the Institutional ethical committee before the start of the study. All patients undergoing caesarean section with excellent dating by first trimester ultrasound were included in the study.

\section{Inclusion criteria}

- Patients undergoing caesarean section who fulfilled the criteria given below were included in this study.

- Both elective and emergency cases were included in the study.

- Only patients with excellent dating with a scan done between 7-12 weeks were taken in this study.

\section{Exclusion criteria}

- Patients with uncertain dates,

- Patients with premature rupture of membranes undergoing section

- All caesarean sections where amniotic fluid was meconium stained

- Cases where amniotic fluid was blood stained.

During caesarean section after uterine incision, initially $5 \mathrm{ml}$ of amniotic fluid was collected and sent to hematology laboratory where amniotic fluid was analysed in platelet counting chamber. No centrifugation was done. Lamellar bodies are of the same size as platelets and hence counted using Coulter hematological analyser. Lamellar Body counts at different gestational ages were noted. Incidence of RDS in newborn babies was noted. The data was statistically analysed. The incidence of RDS at different LBC count was analysed. Cut off of 40,500 was derived after comparing sensitivities and specificities of various cut offs and constructing ROC curve (Figure 1).



Figure 1: ROC curve with LBC cut off of 40,500 .

Respiratory distress syndrome (RDS) in newborn was defined as Oxygen dependence increasing during the first 24 hours of life after excluding infection. Typical chest X ray pattern with reduced air content, reticulonodular pattern of the lung and air bronchogram. An attempt has been made to correlate LBC count with RDS in newborn. A ROC curve was plotted and a LBC count of 40,500 (Figure 1) was taken as cutoff for prediction of RDS in Newborn.

\section{Statistical analysis}

Statistical package for the social science (SPSS-16) was used for statistical compilation and analysis. For statistical analysis chi square test was used when appropriate. Statistical significance was accepted at $\mathrm{P}<$ 0.05 .

\section{RESULTS}

This was a prospective observational study for the period of 1 year in the department of Obstetrics and Gynecology, in a tertiary care hospital. Total of 1020 caesarean deliveries were done among them, 300 patients who fulfilled criteria were included in the study. other 
cases were not included in view of PROM, meconium stained liquor.

Among 300 cases ,160 were term and 140 were preterm. RDS was seen in 116 newborns in the preterm group. Term group had no case of RDS. After constructing ROC (Figure 1), LBC count off of 40,500 was chosen as cutoff as it had sensitivity of $92.7 \%$ and specificity of $90 \%$ (Figure 1). In present study RDS was seen only in preterm fetuses. Majority of the patients belonged to 21 30 -year age group (Table 1). No significant differences in LBCs were noted with regards to parity and age of the patients (Table 1).

Table 1: Age wise, parity distribution of subjects.

\begin{tabular}{|l|l|l|l|}
\hline \multirow{2}{*}{ Age } & Nulliparous & Multiparous & $\begin{array}{l}\text { p } \\
\text { value }\end{array}$ \\
\hline < 20 years (10) & 10 & 0 & 0.4 \\
\hline 21-30 years (192) & 89 & 103 & \\
\hline$>$ 30 years (98) & 35 & 63 & \\
\hline
\end{tabular}

Table 2 tells us about the relation between gestational age and lamellar body counts. Among 140 preterm fetuses, 121 fetuses $(86.8 \%)$ of the fetuses showed LBC counts less than 40,500 and 19 fetuses (13.5\%) of the fetuses had LBC more than 405000 (Table 2).

Table 2: Relation between gestational age and lamellar body count.

\begin{tabular}{|c|c|c|c|}
\hline \multirow[b]{2}{*}{ Gestational age } & \multicolumn{2}{|c|}{ Lamellar body counts } & \multirow[b]{2}{*}{ p value } \\
\hline & $\begin{array}{l}<40,500 \\
\mathrm{~N}=133(\%)\end{array}$ & $\begin{array}{l}>40,500 \\
\mathrm{~N}=167(\%)\end{array}$ & \\
\hline Preterm (140) & $121(86.8)$ & $19(13.5)$ & \multirow{2}{*}{$0.000^{*}$} \\
\hline Term (160) & $12(7.05)$ & $148(92.5)$ & \\
\hline
\end{tabular}

Out of total 160 term fetuses $148(92.5 \%)$ of them had LBC more than 40,500 and only $12(7.05 \%)$ had LBC less than 40500 (Table 2).

The observations noted were significant $(\mathrm{p}<0.0001)$. As gestational age increased Lamellar body counts increased indicating that Lamellar body counts directly proportional to the gestational age. Incidence of RDS in the above studies fetuses was noted (Table 3).

Table 3: Relation between $\mathrm{LBC}$ and RDS, N=300.

\begin{tabular}{|l|l|l|l|}
\hline \multicolumn{1}{|c|}{ RDS } & \multicolumn{3}{|c|}{ LBC } \\
& $\mathrm{N}=133(\%)$ & $\mathrm{N}=167(\%)$ & \\
\hline Yes (116) & $111(83.4)$ & $5(3.4)$ & \multirow{2}{*}{$0.000^{*}$} \\
\hline No (184) & $22(16.5)$ & $161(96.4)$ & \\
\hline
\end{tabular}

Out of 133 fetuses who had reduced lamellar body count $111(83.4 \%)$ fetuses showed signs of RDS. When LBC was more than 40500 , only 5 fetuses had RDS. When LBC count was less than 40,500 the incidence of RDS was seen in $83.4 \%$ of the fetuses with $p$ value being significant. (Table 3). Incidence of RDS reduced significantly as low as $3.4 \%$ when LBC counts were greater (Table 3 ). Increasing lamellar body counts tends to reduce the incidence of RDS in newborns.

\section{DISCUSSION}

Though there are many methods to predict lung maturity in fetus, most of them involve complex labour and are expensive. ${ }^{5} \mathrm{We}$ in our current study tried to correlate the Lamellar Body Concentrations (LBC) at different gestational ages and its role in the prediction of RDS. LBC count can be done easily in auto analyzers as it has same size, that of platelets. In present study we enrolled total of 300 patients who were undergoing caesarean section. Amniotic fluid was collected after uterine incision and sent to the laboratory to calculate LBC in automated hematological analyzer.

In present study we calculated a cut off of 40500 after constructing the ROC curve (Figure 1). LBC count cut off 22,000-57,000 has been suggested by various investigators. ${ }^{6}$ We used a cut off of 40,500 for LBC and found a sensitivity of $92.7 \%$, Specificity of $90 \%$ and positive predictive value of $73 \%$ and negative predictive value of $98 \%$ in predicting RDS. Gary E Ross et al in a study, compared lamellar body counts with lecithin/ sphingomyelin ratio and they concluded that lamellar body count can accurately predict lung maturity when compared with L/S ratio. ${ }^{7}$ The sensitivity and specificity of lamellar body counts in his study was on par with L/S ratio in predicting fetal lung maturity. Roiz-Herna'ndez et al suggested a cut off 57,000, and in his study, he collected samples of amniotic fluid both during caesarean section and vaginal delivery. ${ }^{8}$ In case of vaginal delivery amniotic fluid was immediately collected from posterior fornix after rupture of membranes, and blood stained, and meconium stained liquors were discarded from the study. However, in present study amniotic fluid was collected only during caesarean section to avoid the chances of contamination and possibility of false positive results. Haymond et al compared LBC count with fluorescent polarization of amniotic fluid and found that LBC counts were on par with fluorescent polarization of amniotic fluid in predicting RDS in preterm fetuses. ${ }^{9}$

In a study by Khazardoost S et al LBC cut off of 50000 was taken. He found a sensitivity and specificity of $85 \%$ and $70 \%$ respectively in predicting $\operatorname{RDS}^{6}$ Chapman et al used a cut off of 35,000 . He also used different auto analyzers and found that there was no difference in LBC counts when different auto analyzers were used. ${ }^{10}$ Chapman et al found a sensitivity of $100 \%$ and specificity of $68 \% 10$. Bahasadri et al used cut off of 45000 and concluded that LBC counts above 45000 predicts lung maturity with sensitivity and specificity of $99 \%$ and $98 \%$ respectively. ${ }^{11} \mathrm{He}$ also found that when LBC counts were less than 10,000 there was always RDS in newborn infants. In the current study, there were 140 preterm 
babies. 121 babies had count less than 40,500. Among these 116 had RDS. The incidence of RDS sharply fell when the LBC count $>40,500$. Only 5 babies out of 15 had RDS when LBC count was more than 40,500. Thus, LBC count correlates with the directly with the increasing gestational age, greater the gestational age more is the LBC count and this in turn is inversely proportional to the incidence of RDS in preterm babies.

Thus, this test is non-expensive test which can be done easily in smaller settings for prediction of RDS in new born fetus especially in developing countries. Thus, LBC count can be suggested as a method of assessing fetal lung maturity when delivery has to be planned for preterm or in cases of pregnancies with unknown dates.

In future whenever preterm delivery is anticipated, Amniotic fluid can be obtained by amniocentesis and LBC counts can be analyzed. The decision to deliver or prolong the pregnancy could be made based on the fetal lung maturity. If the pregnancy has to be terminated at preterm gestational age for underlying condition like preeclampsia, then amniotic fluid sample LBC can be done by amniocentesis and if fetal lung immaturity is detected then those cases can be referred to tertiary care for delivery where facilities of NICU care are available to treat RDS. It may be useful for unbooked cases or for previous caesarean cases who come in 3rd trimester with poor dating. Ultrasound in the third trimester is good for fetal growth and wellbeing and not for dating. Often there is dilemma to time the elective caesareans in cases with poor dating. $\mathrm{LBC}$ is thus a simple method to assess fetal lung maturity which is useful in clinical practice.

Thus, LBC count can serve as a reliable and an easy method of assessing fetal lung maturity and predicting RDS in newborns.

\section{CONCLUSION}

\section{ACKNOWLEDGMENTS}

Authors would like to thank Dr Chetan Manohar from Department of Pathology, for helping in estimation of lamellar body in the amniotic fluid sample. Dr Vasudev, Department of Statistics, SDM Dharwad for analysis.

\section{Funding: No funding sources} Conflict of interest: None declared

Ethical approval: The study was approved by the Institutional Ethics Committee

\section{REFERENCES}

1. Angus D, Linde-Zwirble W, Clermont G, Griffin MF, Clark RH. Epidemiology of neonatal respiratory failure in the United States: Projections from California and NewYork. Am J Respir Crit Care Med. 2001;164(7):1154-60.

2. Fakhoury G, Daikoku NH, Benser J, Dubin NH. Lamellar body concentrations and the prediction of fetal pulmonary maturity. Am J Obstet Gynecol. 1994;170(1):72-6

3. Lafler DJ, Mendoza A. Laboratory testing to assess fetal lung maturity. Lab Med. 2001;32(7):393-6.

4. Smith BT. Fibroblast -Pnuemocyte factor: Am J Physiol Lung Cell Mol Physiol 1989;257(4):174-8

5. Grenache DG, GronowskiAM. Fetal lung maturity. Clin Biochem.2006;39(1):1-10.

6. Khazardoost S, Yahyazadeh H, Borna S, Sohrabvand F, Yahyazadeh N, Amini E. Amniotic fluid lamellar body count and its sensitivity and specificity in evaluating of fetal lung maturity: J Obstet Gynaecol. 2005;25(3):257-9

7. Ross GE, Bever FN, Uddin Z, Hockman EM, Herman BA. Decreased laboratory testing for lecithin-to-sphingomyelin ratio and phosphatidylglycerol after fetal lung maturity assessment from lamellar body count in amniotic fluid. JAOA. 2002;102(8):8-14

8. Roiz-Hernández J, Navarro-Solis E, Carreón-Valdéz E. Lamellar bodies as a diagnostic test of fetal lung maturity. Int J Gynecol Obstet. 2002;77(3):217-21.

9. Haymond S, Luzzi VI, Parvin CA,Gronowski AM. A direct comparison between lamellar body counts and fluorescent polarization methods for predicting respiratory distress syndrome.Am J ClinPathol.2006;126(6):894-9.

10. Chapman JF, Ashwood ER, Feld R, Wu AH. Evaluation of two-dimensional cytometric lamellar body counts on the ADVIA ${ }^{\circledR} 120$ hematology system for estimation of fetal lung maturation. Clinica Chimica Acta. 2004;340(1-2):85-92.

11. Bahasadri S, Changizi N. Association between lamellar body count and respiratory distress in neonates. Saudi Med J. 2005;26(9):1414-6.

Cite this article as: Amruta CC, Patil HA.

Correlation between amniotic fluid lamellar body concentrations at different gestational ages and respiratory distress syndrome. Int J Reprod Contracept Obstet Gynecol 2019;8:188-91. 